\title{
Investigations of orientation and length scale effects on micromechanical responses in polycrystalline Zirconium using spherical nanoindentation
}

\author{
Siddhartha Pathak ${ }^{1,2^{*}}$, Surya R. Kalidindi ${ }^{3}$, Nathan A. Mara ${ }^{1,4^{*}}$
}

${ }^{1}$ Center for Integrated Nanotechnologies, Los Alamos National Laboratory, Los Alamos, NM

${ }^{2}$ Chemical and Materials Engineering, University of Nevada, Reno, NV

${ }^{3}$ George W. Woodruff School of Mechanical Engineering, Georgia Institute of Technology, Atlanta, GA

${ }^{4}$ Institute for Materials Science, Los Alamos National Laboratory, Los Alamos, NM

*corresponding author: siddharthapathak@gmail.com, namara@lanl.gov; 505-667-8665, PO Box 1663, MS-K771, Los Alamos, New Mexico, 87545 USA

\begin{abstract}
Here we investigate the elastic and plastic anisotropy of hexagonal materialsas a function of crystal orientation using a high-throughput approach (spherical nanoindentation). Using high purity zirconium as a specific example, we demonstrate the differences in indentation moduli, indentation yield strengths and indentation post-elastic hardening rates over multiple grain orientations. These results are validated against bulk single crystal measurements, as well as data from cubic materials. By varying the indenter size (radius), we are also able to demonstrate indentation size effects in
\end{abstract}


hexagonal materials, including possible signatures of strain hardening due to twin formation in the nanoindentation stress-strain curves.

Keywords: Twinning; nanoindentation; electron backscattering diffraction (EBSD); work hardening; indentation stress-strain

\section{Text:}

Nanomechanical testing techniques, such as nanoindentation [1-3], micropillar compression and tension $[4,5]$, as well as three point bend and cantilever bend techniques [6-8], have been used for the past couple of decades to probe small volumes of material of the order of $1 \mu \mathrm{m}^{3}$ or smaller. In techniques other than nanoindentation, preparation of miniaturized tensile and compression samples is typically carried out in bulk materials using Focused Ion Beam (FIB) milling, a time consuming technique that runs the risk of ion beam damage to the sample $[9,10]$. Nanoindentation, on the other hand, provides copious amounts of mechanical data from extremely small volumes of material while only requiring a flat polished surface[11]. Much work has also been carried out over the past few years to obtain stress-strain curves using a spherical nanoindentation approach $[2,11-13]$. This technique can transform the raw loaddisplacement data into meaningful indentation stress-strain curves that capture the local loading and unloading elastic moduli, local indentation yield strengths, and post-yield strain hardening behavior.As such, spherical nanoindentation represents a highthroughput technique that can amass enormous amounts of grain-level data from one polycrystalline sample.

Early work utilizing this technique focused largely on orientation dependent local (grain-scale) mechanical responses in relatively low anisotropy cubic systems such as 
face centered cubic (fcc) Aluminum and body centered cubic (bcc) Tungsten[2, 12, 14]. More recent studies have explored more elastically anisotropic cubic materials such as Fe-3\%Si steel $[15,16]$,thework-hardening in grain-boundary regions in polycrystalline metallic samples [17-19], and the influence of ion implantation on damage gradients relative to the sample surface in Tungsten[13].In this work we report on the use of our recently developed spherical nanoindentation stress-strain techniques on materials with non-cubic crystal structure that deform extensively via twinning. While nanoindentation of hexagonal materials have been previously reported using both pyramidal[20] and spherical indenters[21, 22], the use of indentation stress-strain analysis is advantageous in that it allows us to study not only the commonly reported modulus and hardness values in these materials, but also their yield strengths under indentation, as well as their post yield hardening response and how the indentation length scale (controlled by changing the indenter tip radius) affects their propensity of twinning under the indenter.

Hexagonal close packed (hcp) metals such as zirconium (Zr) represent increasing complexity in mechanical behavior when compared to the relatively symmetric slip inherent to cubic systems. Zirconium's mechanical behaviorhas been studied extensively, and is known to be highly anisotropic compared to cubic metalsshowing varying degrees of dislocation slip and twinning depending upon loading direction[23-26].In this vein, polycrystalline $\mathrm{Zr}$ is the ideal test bed for a spherical nanoindentation investigation into microscale mechanical anisotropy. The current work will leverage modern highthroughput nanomechanical test techniques to determine differently oriented single crystal stress-strain responses of $\mathrm{Zr}$ at different length scales (by varying the indenter tip radius; all length scales studied here are significantly smaller than the millimeter sized 
grains in the sample). Note that in the current study only three carefully polished samples of $\mathrm{Zr}$ will be required to explore the complete range of orientations in hcp $\mathrm{Zr}$ (see Fig. 1), in comparison to the dozens of painstakingly prepared single crystal samples needed by past researchers.

In this study, spherical nanoindentations with different indenter tip radii were conducted on $\sim 50$ grains distributed in three samples[27](see Supporting Information section). Indentation stress-strain curves were computed from the corresponding raw load-displacement datasets following the procedures outlined in Eqs. S1 and S2[11, 12].The results from fourrepresentative grains, noted here as Grains $1,2,3$, and 4, which have sufficiently differing crystallographic orientations measured using electron backscatter diffraction (EBSD) that are spread over the fundamental orientation zone (depicted as the inverse pole figure (IPF) "triangles" in Figure 1) are discussed in detail in this work. The orientation of Grain 1 provides for an indentation direction almost parallel to the crystallographic c-axis, and that of Grains 3 and 4 almost perpendicular to the c-axis, while Grain 2 lies in the center of the IPF triangle. Figure 1d shows a relative comparison of the indentation load-displacement responses measured with a $10 \mu \mathrm{m}$ indenter radius between these four grains. As expected, Grain 1 shows the strongest response, as it invokes the hardest deformation modes available in $\mathrm{Zr}$.

In Figure 2, the extracted indentation stress-strain curves for Grain 1 are shown for spherical indenters ranging in radii from $10 \mu \mathrm{m}$ to $100 \mu \mathrm{m}$. For the larger indenter sizes (of radius $100 \mu \mathrm{m}$ ), no pop-ins are evident, and the indentation yield stress ( $Y_{\text {ind }}$, measured as a $0.2 \%$ offset stress) is $1279.7 \pm 151 \mathrm{MPa}$ (see Table 1). For the smaller $10 \mu \mathrm{m}$ radiusindenter, there is typically a large pop-in event (sometimes followed by several 
smaller pop-ins), and a subsequent steady-state work hardening at a rate of 11.2 $\pm 0.7 \mathrm{GPa}$. The work hardening rate was calculated as the linear slope of the indentation stress-strain curve between offset strains of 1 and $2 \%$.

Figure 3 shows the corresponding indentation stress-strain responses for Grains 2, 3 and 4 measured with five different indenters of $1,5,10,100$ and $150 \mu \mathrm{m}$ radii. These grain orientations, which range from indentation perpendicular to the c-axis (Grains 3,4) to another off-axis orientation (Grain 2) exhibit very different behavior than that found in the tests parallel to the c-axis (Grain 1). In contrast to the c-axis grains, Grains 2, 3 and 4 show distinct length scale effects, which can be broadly grouped in three general patterns. (i) Tests with the smallest sized indenter ( $1 \mu \mathrm{m}$ radius $)$ show a series of pop-ins that continue for the entire duration of the test (until a strain of over 0.25, green curves in Fig. $3)$. This phenomena is commonly referred to as 'staircase yielding' [20, 28, 29] for loadcontrolled indentations, where the indentation strain (or depth) excursions correspond to discrete plastic deformation events and are separated by loading portions that are predominantly elastic[14, 15, 30]. (ii) At intermediate indenter sizes $(5 \mu \mathrm{m}$ and $10 \mu \mathrm{m}$ radii indenters) the series of pop-ins continues up to a critical strain level, and is then followed by rapid work hardening in the indentation stress-strain response. The strains at which the steady-state work hardening begins is seen to decrease with increasing indenter size. Thus for the $5 \mu \mathrm{m}$ radius indenter Grains 2, 3 and 4 show repeated pop-ins up to indentation strains of $0.064 \pm 0.003,0.078 \pm 0.005$ and $0.081 \pm 0.007$, while the same strain values for the $10 \mu \mathrm{m}$ radius indenter are lower at $0.055 \pm 0.005,0.06 \pm 0.005$ and $0.058 \pm 0.008$ respectively, whereupon steady-state work hardening with no pop-in ensues. Table 2 summarizes the measured contact radius (a) values (Eq. S2) at these 
corresponding indentation strain levels. (iii) For the largest indenter sizes (100 and 150 $\mu \mathrm{m}$ radii indenters), pop-ins are typically small or entirely absent. Thus the data collected with the largest $150 \mu \mathrm{m}$ radius indenter is ideal for measuring $Y_{\text {ind }}$ (see Table 1) since the absence of pop-ins makes it easier to discern the yield point for these indenter sizes.As seen from Table 1, the $Y_{\text {ind }}$ values for Grains 2, 3 and 4 are nearly an order of magnitude lower than that seen in c-axis compression (Grain 1).

Plastically, zirconium has been found to deform in three slip modes, prismatic $<\mathrm{a}>$, pyramidal $<\mathrm{c}+\mathrm{a}>$, and basal $<\mathrm{a}>$. Also possible are two different types each of extension twinning and contraction twinning, $\{10-12\}(10-1-1)$ and $\{11-21\}(11-2-6)$ (extension) and $\{11-22\}(11-2-3)$ and $\{10-11\}(10-1-2)$ (contraction). Studies by Akhtar et al.[23-26] have shown that at room temperature, prismatic slip is expected to be the easiest slip pathway for orientations where applied resolved shear stresses are favorable. However, in compression along the $<\mathrm{c}>$ axis, where prismatic slip is not favored, $\mathrm{Zr}$ crystals deform preferentially by $\{11-22\}$ contraction twinning and increasingly by pyramidal $<\mathrm{c}+\mathrm{a}>$ slip with increasing temperature. Similarly Beyerlein and Tome [ 31 , 32] have shown that in high purity polycrystalline $\mathrm{Zr}$ (average grain size $25 \mu \mathrm{m}$ [33])at $300 \mathrm{~K}$, subjected tocompression along the c-axis such as is the case for Grain 1, pyramidal slip is preferred,and the corresponding slip resistances are an order of magnitude higher than those found for prismatic slip.

The above discussion can help explain the observations from Figs. 2-3 and Tables 1 and 2. As seen from Fig. 2, the indentation on Grain 1 forces a contraction of the c-axis. For such an imposed deformation on the crystal, there is usually no resolved shear stress on the prismatic $<\mathrm{a}>$ slip systems, and so the material is forced to deform by other hard 
modes (contraction twinning and/or pyramidal $<\mathrm{c}+\mathrm{a}>$ ). This is reflected in the high indentation yield stresses for Grain 1 as compared to thosemeasured for Grains 2, 3 and 4. The ratio of $Y_{\text {ind }}$ values for Grain 1 with respect to the other 3 grains(see Table 1) is $\sim 11-12$, which is in good agreement with the ratio of the hard/soft deformation mode (i.e., slip and twin) resistances used by Beyerlein and Tome[31]. In fact, for our study, we used material from the same melt of high-purity $\mathrm{Zr}$, so a strong correlation is expected, and is anindicator of the validity of our high-throughput technique. Similarly the elastic modulus data shown in Table 1 also matches well with the trends seen in bulk single crystal measurements for the orientations measured [34], within 5-15\% of those values found in bulk crystals.

Beyond the measures of yield stress and elastic modulus, the indentation stressstrain technique as applied here can also be employed to investigate post-yield hardening behavior. Note that most commonly used indentation protocols $[1,3,35]$ are unable to resolve the early stages of the stress-strain curves (elastic and elastic-plastic transitions) and therefore are incapable of providing the insights obtained and presented here. Table 1 compares the indentation results for the current work on hep $\mathrm{Zr}$ to two cubic materials, bcc Tantalum and fcc Aluminum. Note the high $Y_{\text {ind }}$ value, as well as the high post-yield normalized strain hardening rate of Grain 1 in $\mathrm{Zr}$ as compared to the other materials (five times higher than that exhibited by Tantalum or Aluminum). Even for $\mathrm{Zr}$ oriented favorably for prismatic slip (Grains 2, 3 and 4), the normalized work hardening rate is higher than that for Tantalum or Aluminum. All three metals included in this comparison ( $\mathrm{Al}, \mathrm{Ta}$, and pure $\mathrm{Zr}$ ) are all high stacking fault energy materials; consequently these differences are not merely differences in stage II hardening rates. 
The physical origin of the high hardening rates in $\mathrm{Zr}$ (compared to $\mathrm{Al}$ or Ta)can be attributed to a number of factors, and is possibly likely to be very different for the differently orientation grains studied here. For Grain 1, the high hardening rates (the highest measured in our experiments) are possibly a consequence of the activation of the hard deformation modes (pyramidal $<\mathrm{c}+\mathrm{a}>$ slip and some degree of contraction twinning).Such high strain hardening rates have indeed been reported in bulk testing of $\mathrm{Zr}$ at room temperature[23-26] as well as in other hep metals exhibiting hard deformation modes[36-39]. In particular, we point out that the activation of deformation twinning should transform the initially single crystal region in the indentation zone to a polycrystalline microstructure, which can then lead to high hardening rates via a HallPetch mechanism[36-39]. We also note that the production of twins essentially transforms glissile dislocations to sessile dislocations (also called the Basinski mechanism[40]), which can also serve as a potent strain hardening mechanism. Clearly, these observations call for detailedmicroscopy investigations of deformed regions under the indenter in order to identify the responsible physical mechanisms.

The strain hardening rates observed in the indentations on Grains 2, 3 and 4 are lower than those observed in Grain 1 (Table 1). This is because the expected deformation modes in grains 2,3 , and 4 are mainly prismatic $<a>$ slip and extension twins. Note that the stress needed to produce an extension twin in room temperature deformation of $\mathrm{Zr}$ is substantially lower than that required to activate either pyramidal $<\mathrm{c}+\mathrm{a}>$ slip and/or contraction twins[23-26]. Consequently, Grains 2, 3 and 4 represent softer orientations compared to Grain 1. However, we also expect the stress required to activate extension twinning to be somewhat higher than the stress needed to activate prismatic $<a>$ slip. So 
we might expect Grain 2 to exhibit a lower indentation yield stress compared to Grains 3 and 4. This is indeed reflected in our measurements (see Table 1). This difference is somewhat mitigated in our experiments because of two reasons: (i) While prismatic $<\mathrm{a}>$ slip is likely to be more prevalent in the indentation of Grain 2, all three grains exhibit both prismatic $<\mathrm{a}>$ slip and extension twinning as the former alone is insufficient to accommodate the complex deformation mode imposed in the indentation zone. This is also evident in the slip and twin markings visible on the SEM micrographs of the deformed indentations shown in Fig. 3a-c.(ii) Although extension twinning is demanded to accommodate the extension in the c-axis direction in the highly heterogeneous indentation zone in these experiments, this driving force is somewhat diminished by the fact that the c-axis is at a large angle with respect to the indentation direction in Grains 2, 3 and 4 (unlike Grain 1 where the c-axis is forced to contract without exception). Consequently, the driving force for extension twinning is rather mitigated by the anisotropic response in the indentation zone (i.e., the other directions expanding more compared to the relatively hard extension of the c-axis). This is clearly evident in the anisotropic geometries of the residual indentations on the sample in Grains 2, 3 and 4 compared to the symmetric (circular) residual imprint on Grain 1 (comparing SEM micrographs in Figs. 2 and 3).

The study presented here has also revealed several new insights into length scale effects. In particular, the influence of the length scale (controlled by indenter tip radius) is seen to be most dramatic on the elastic-plastic transition. One of the effects known from prior studies $[14,15,18]$ is that the propensity for observing pop-in eventson annealed samples increases with smaller indenter tips. This, of course, is also clearly seen in the 
results presented in this study. Additionally, we observe several additional length scale effects in the indentation measurements reported here on hcp metals that exhibit deformation twinning in addition to crystallographic slip[41]. At the outset, it is important to note that both slip and twin resistances are expected to exhibit strong dependencies on length scales in addition to crystallographic orientation[42]. In general, the extent of deformation twinning should be expected to be higher in the indentation experiments performed with the larger indenter tips. As noted earlier, activation of deformation twinning should result in high hardening rates because of the Hall-Petch and Basinski mechanisms mentioned earlier. Figure 3 and Table 2support this hypothesis through the observation that the measurements with the smallest $1 \mu \mathrm{m}$ radius indenter tip do not attain high hardening rates even at large plastic strains, unlike the measurements with the larger indenters on the same grains. This is because the volumes explored with the smaller indenters are substantially smaller, requiring significantly larger stresses to nucleate deformation twins. The zone under the indenter undergoing plastic deformation is estimated to correspond approximately to a cylinder of radius $a$ and height $2.4 a[43$, 44]. As seen from Table 2 for the $1 \mu \mathrm{m}$ indenter radius case in Grain 2, the maximum value of $a$ at the end of the test is $609 \pm 3 \mathrm{~nm}$, which is considerably smaller than the $a$ values attained with the larger indenter sizes (of radii 5 and $10 \mu \mathrm{m}$ ) at the onset of hardening. Similar trends can be seen for Grains 3 and 4 as well. This implies that once a sufficient volume of material undergoes plastic deformation beneath the indenter, possibly by a combination of multiple slip and twin modes, it consistently leads to a high rate of work hardening in Zr. Thus, for the 5 and $10 \mu \mathrm{m}$ indenter radii cases, the stressstrain curves in Fig. 3 indicate that the elastic response is followed by discrete plastic 
deformation events (either deformation twinning and/or dislocation slip depending on the crystal orientation) until sufficient sources are activated to enable multiple modes. This is then followed by a uniform work hardening exceeding those previously seen in literature for cubic material systems. For the largest indenter sizes (100 and $150 \mu \mathrm{m}$ radii indenters), hardening starts immediately after yield, since $a$ has already reached a very large value (of $\sim 1.3 \mu \mathrm{m}$ at yield).

Thus the conditions in the tests with the smaller indenters appear to be such that the desired critical length (volume)scales for activation and/or growth of deformation twinshave not been met.

In conclusion, a high throughput methodology based on spherical nanoindentation stress-strain analysis shows efficacy in determining the elastic and plastic anisotropy of high purity Zr. Through the use of only three metallographically prepared samples, the mechanical behavior of $\mathrm{Zr}$ compressed perpendicular and parallel to the c-axis were shown to have close correlation to bulk single crystal measurements. An indentation size effect was revealed, whereby a critical deformation volume was found to be necessary to facilitate steady state work hardening for grains compressed in off c-axis orientations.

\section{Acknowledgements:}

The authors thank Dr. Ellen K. Cerretaand Dr. Rodney J. Mccabe (LANL) for help with sample preparation. The authors gratefully acknowledge support from the U.S. Department of Energy, Office of Nuclear Engineering, Nuclear Engineering Enabling Technologies (DOE-NEET), as well as fruitful discussions with Dr. Irene Beyerlein. This work was performed, in part, at the Center for Integrated Nanotechnologies, an Office of Science User Facility operated for the U.S. Department of Energy (DOE) Office of 
Scripta Materialia

Science. Los Alamos National Laboratory, an affirmative action equal opportunity employer, is operated by Los Alamos National Security, LLC, for the National Nuclear Security Administration of the U.S. Department of Energy under contract DE-AC5206NA25396.

[1] W.C. Oliver, G.M. Pharr, Journal of Materials Research, 19 (2004) 3-20.

[2] S. Pathak, J. Shaffer, S.R. Kalidindi, Scripta Materialia, 60 (2009) 439-442.

[3] C.A. Schuh, Materials Today, 9 (2006) 32-40.

[4] N. Mara, I. Beyerlein, J Mater Sci, 49 (2014) 6497-6516.

[5] M.D. Uchic, D.M. Dimiduk, J.N. Florando, W.D. Nix, Science, 305 (2004) 986-989.

[6] B.N. Jaya, C. Kirchlechner, G. Dehm, Journal of Materials Research, 30 (2015) 686698.

[7] N.A. Mara, J. Crapps, T.A. Wynn, K.D. Clarke, A. Antoniou, P.O. Dickerson, D.E. Dombrowski, B. Mihaila, Philosophical Magazine, 93 (2013) 2749-2758.

[8] K.J. Hollis, N.A. Mara, R.D. Field, T.A. Wynn, J.M. Crapps, P.O. Dickerson, J Therm Spray Tech, 22 (2013) 233-241.

[9] J.R. Greer, J.T.M. De Hosson, Progress in Materials Science, 56 (2011) 654-724.

[10] D. Kiener, C. Motz, M. Rester, M. Jenko, G. Dehm, Materials Science \& Engineering A 459 (2007) 262-272.

[11] S. Pathak, S.R. Kalidindi, Materials Science and Engineering: R: Reports, 91 (2015) $1-36$.

[12] S.R. Kalidindi, S. Pathak, Acta Materialia, 56 (2008) 3523-3532.

[13] S. Pathak, S.R. Kalidindi, Y. Wang, R. Doerner, N. A.Mara, submitted, (2015).

[14] S. Pathak, J.L. Riesterer, S.R. Kalidindi, J. Michler, Applied Physics Letters, 105 (2014) 161913.

[15] S. Pathak, D. Stojakovic, R. Doherty, S.R. Kalidindi, Journal of Materials Research Focus Issue on Indentation Methods in Advanced Materials Research, 24 (2009) 11421155.

[16] S. Pathak, D. Stojakovic, S.R. Kalidindi, Acta Materialia, 57 (2009) 3020-3028.

[17] S.R. Kalidindi, S.J. Vachhani, Current Opinion in Solid State and Materials Science, 18 (2014) 196-204.

[18] S. Pathak, J. Michler, K. Wasmer, S.R. Kalidindi, Journal of Materials Science, 47 (2012) 815-823.

[19] S.J. Vachhani, S.R. Kalidindi, Acta Materialia, 90 (2015) 27-36.

[20] Y.T. Pei, G.M. Song, W.G. Sloof, J.T.M. De Hosson, Surface and Coatings Technology, 201 (2007) 6911-6916.

[21] M.W. Barsoum, A. Murugaiah, S.R. Kalidindi, T. Zhen, Y. Gogotsi, Carbon, 42 (2004) 1435-1445. 
[22] A. Murugaiah, M.W. Barsoum, S.R. Kalidindi, T. Zhen, Journal of Materials Research, 19 (2004) 1139-1148.

[23] A. Akhtar, Journal of Nuclear Materials, 47 (1973) 79-86.

[24] A. Akhtar, Acta Metallurgica, 21 (1973) 1-11.

[25] A. Akhtar, Metallurgical Transactions A, 6 (1975) 1217-1222.

[26] A. Akhtar, A. Teghtsoonian, Acta Metallurgica, 19 (1971) 655-663.

[27] G.C. Kaschner, C.N. Tomé, I.J. Beyerlein, S.C. Vogel, D.W. Brown, R.J. McCabe, Acta Materialia, 54 (2006) 2887-2896.

[28] S.G. Corcoran, R.J. Colton, E.T. Lilleodden, W.W. Gerberich, Physical Review B (Condensed Matter), 55 (1997) 16057-16060.

[29] W.W. Gerberich, J.C. Nelson, E.T. Lilleodden, P. Anderson, J.T. Wyrobek, Acta Materialia, 44 (1996) 3585-3598.

[30] D.F. Bahr, D.E. Kramer, W.W. Gerberich, Acta Materialia, 46 (1998) 3605-3617.

[31] I.J. Beyerlein, C.N. Tomé, International Journal of Plasticity, 24 (2008) 867-895.

[32] M. Knezevic, M. Zecevic, I.J. Beyerlein, J.F. Bingert, R.J. McCabe, Acta Materialia, 88 (2015) 55-73.

[33] C.N. Tomé, P.J. Maudlin, R.A. Lebensohn, G.C. Kaschner, Acta Materialia, 49 (2001) 3085-3096.

[34] D. Tromans, International Journal of Research and Reviews in Applied Sciences, 6 (March 2011) 462-483.

[35] W.C. Oliver, G.M. Pharr, Journal of Materials Research, 7 (1992) 1564-1580.

[36] M. Knezevic, A. Levinson, R. Harris, R.K. Mishra, R.D. Doherty, S.R. Kalidindi, Acta Materialia, 58 (2010) 6230-6242.

[37] A.A. Salem, S.R. Kalidindi, R.D. Doherty, S.L. Semiatin, Metall and Mat Trans A, 37 (2006) 259-268.

[38] A. Salem, S.R. Kalidindi, R.D. Doherty, Acta Materialia, 51 (2003).

[39] S.R. Kalidindi, A.A. Salem, R.D. Doherty, Advanced Engineering Materials, 5 (2003) 229-232.

[40] Z.S. Basinski, M.S. Szczerba, M. Niewczas, J.D. Embury, S.J. Basinski, Rev. Metall., 94 (1997) 1037.

[41] R. Sánchez-Martín, M.T. Pérez-Prado, J. Segurado, J.M. Molina-Aldareguia, Acta Materialia, 93 (2015) 114-128.

[42] E. El-Danaf, S. Kalidindi, R. Doherty, Metall and Mat Trans A, 30 (1999) 12231233.

[43] B.R. Donohue, A. Ambrus, S.R. Kalidindi, Acta Materialia, 60 (2012) 3943-3952.

[44] D.K. Patel, H.F. Al-Harbi, S.R. Kalidindi, Acta Materialia, 79 (2014) 108-116.

[45] S.J. Vachhani, N. Mara, V. Livescu, E. Cerreta, in: EPJ Web of Conferences, 2015 accepted. 


\section{Figure and Table captions:}

Figure 1. (a-c) Montage of several EBSD micrographs of three annealed zirconium samples. Note that between the three samples, the entire orientation space for grains in $\mathrm{Zr}$ can be investigated. (d) Comparison of the load-displacement responses between grains 1 to 4 using a $10 \mu \mathrm{m}$ radius spherical indenter.

Figure 2. Spherical nano-indentation stress strain response of Grain 1, measured with two indenters of radii 10 and $100 \mu \mathrm{m}$. Inset: Orientation of Grain 1 on the IPF triangle. Right image: residual imprint from a $100 \mu \mathrm{m}$ radius indenter on Grain 1.

Figure 3. Spherical nano-indentation stress strain responses of (a) Grain 2, (b) Grain 3 and (c) Grain 4. Results from fivedifferent indenters of radii 1, 5, 10, 100 and $150 \mu \mathrm{m}$ are shown for each case. The insets show the orientations of the respective grains on the IPF triangle. The SEM micrographs show the residual imprints from a $100 \mu \mathrm{m}$ radius indenter on each grain. The micrographs were taken at a $52^{\circ}$ tilt angle, and are shown here after correcting for the tilt angle. Due to shadowing effects caused by the high tilt angle, the material pile-ups on the left and right sides of the indents appear as darker regions in the micrographs; the deepest regions of the indent are towards the center of the micrographs. The dotted markings on the sample surfaces are an artifact from the EBSD scanning pattern.

Table 1. Comparison of the indentation loading modulus $\left(E_{\text {eff }}\right)$, the indentation yield $\left(Y_{\text {ind }}\right)$, and hardening rates between the four grain orientations in $\mathrm{Zr}$, and fcc $\mathrm{Al}$ [19] and bcc Ta [45].

Table 2. Comparison of the contact radius ( $a$ ) values measured for Grains 2, 3 and 4 for the five different indenter radii. 

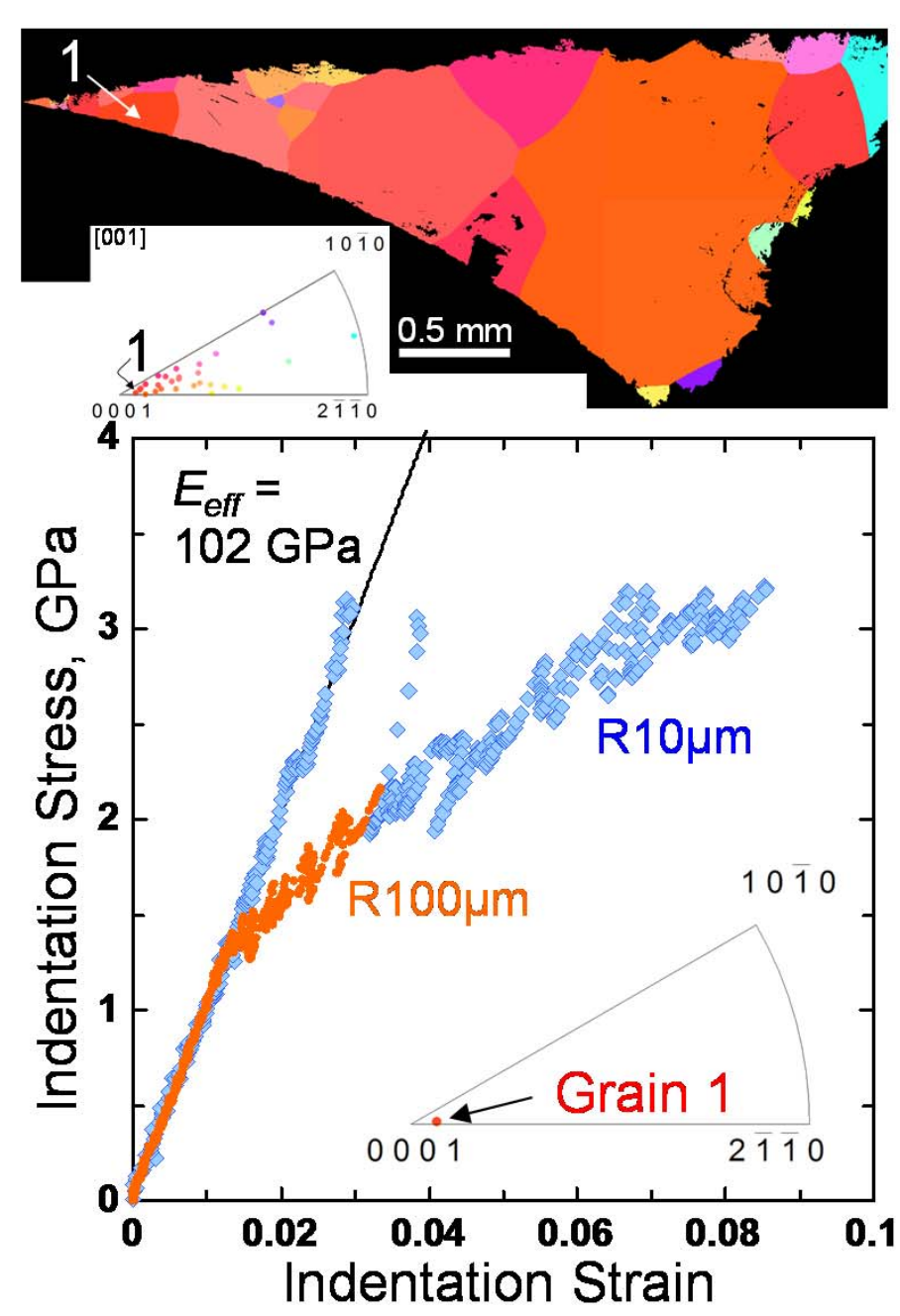

Deformation induced primarily by pyramidal slip
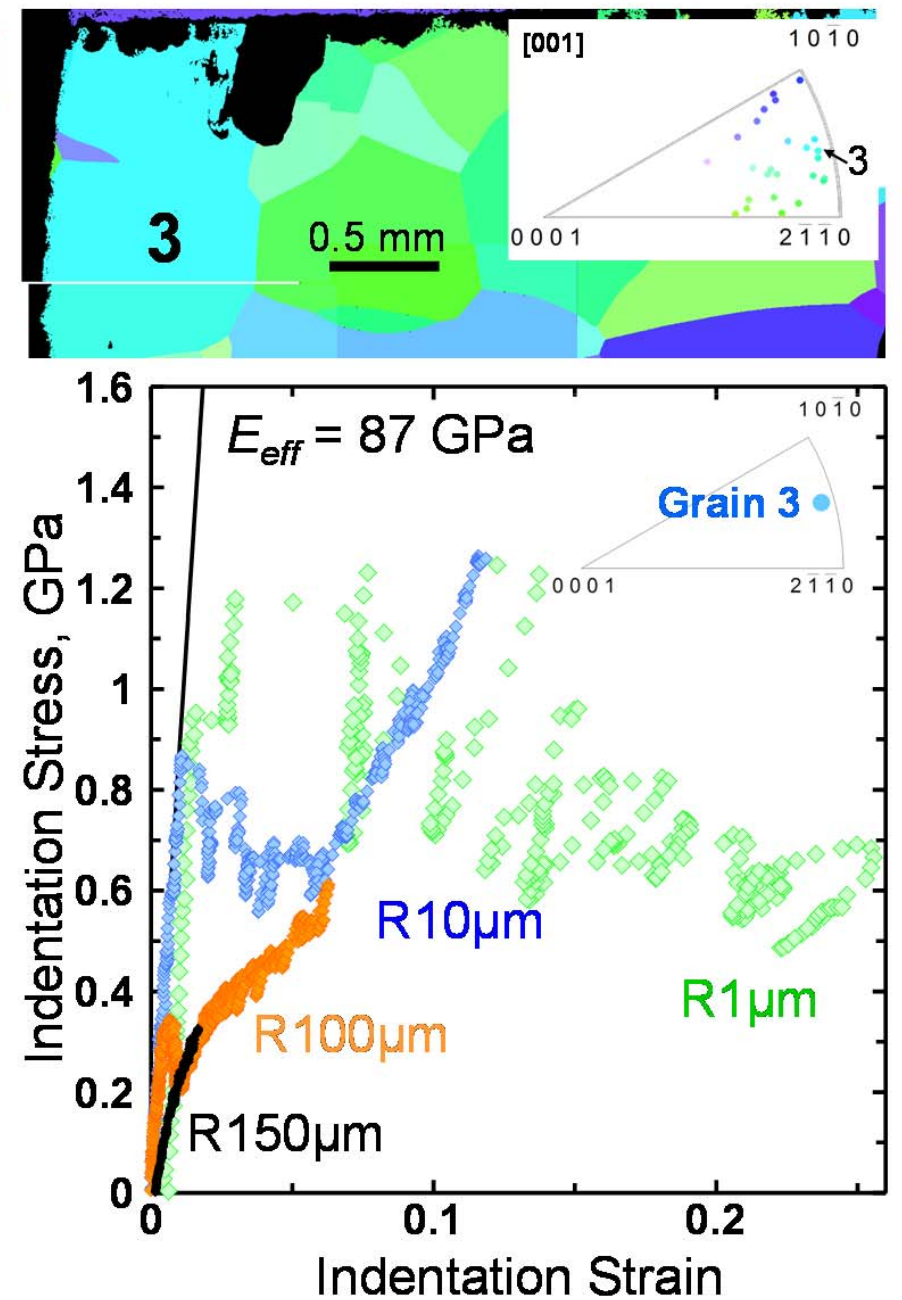

Deformation induced primarily by extension twinning

Graphical abstract 


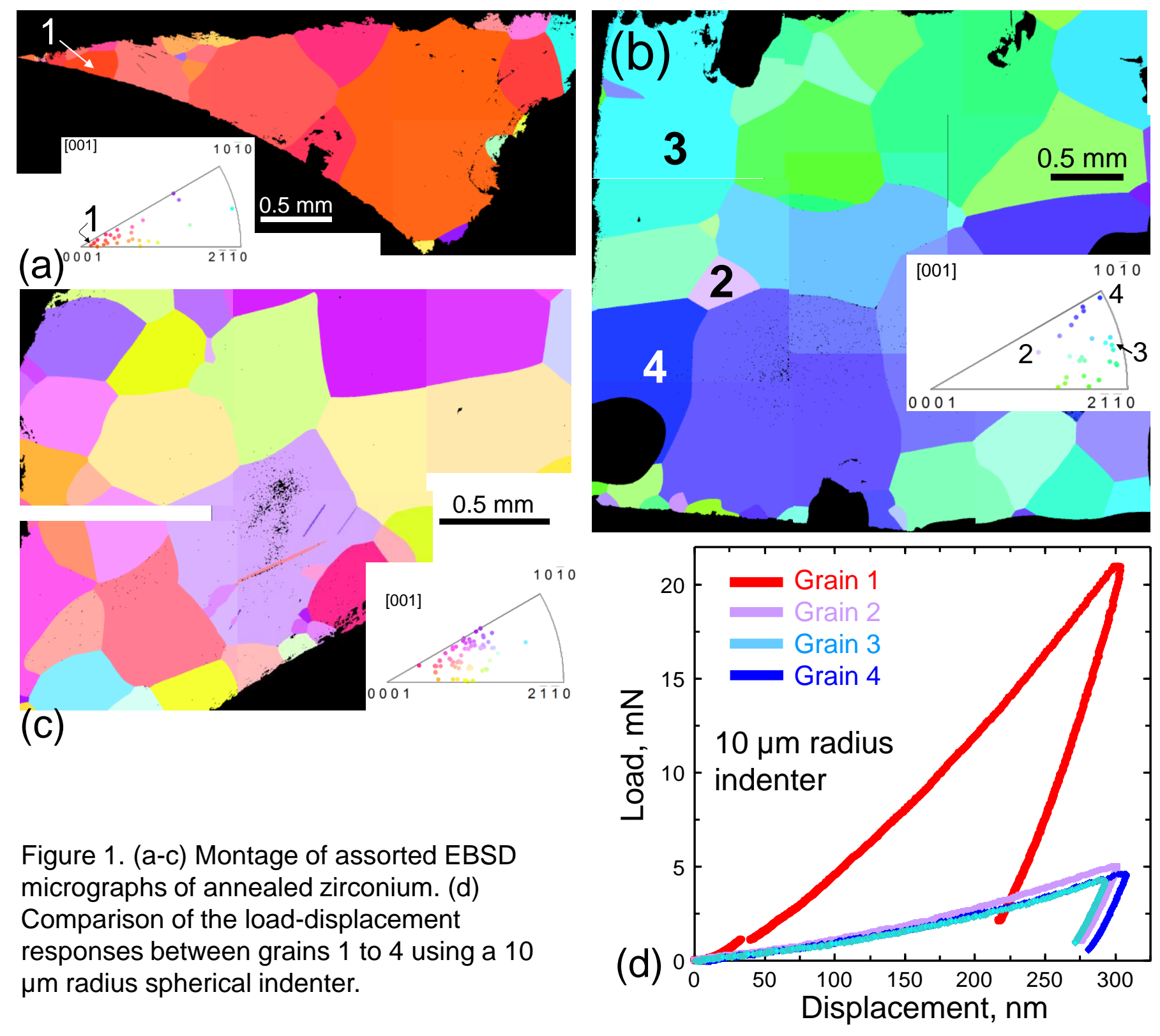




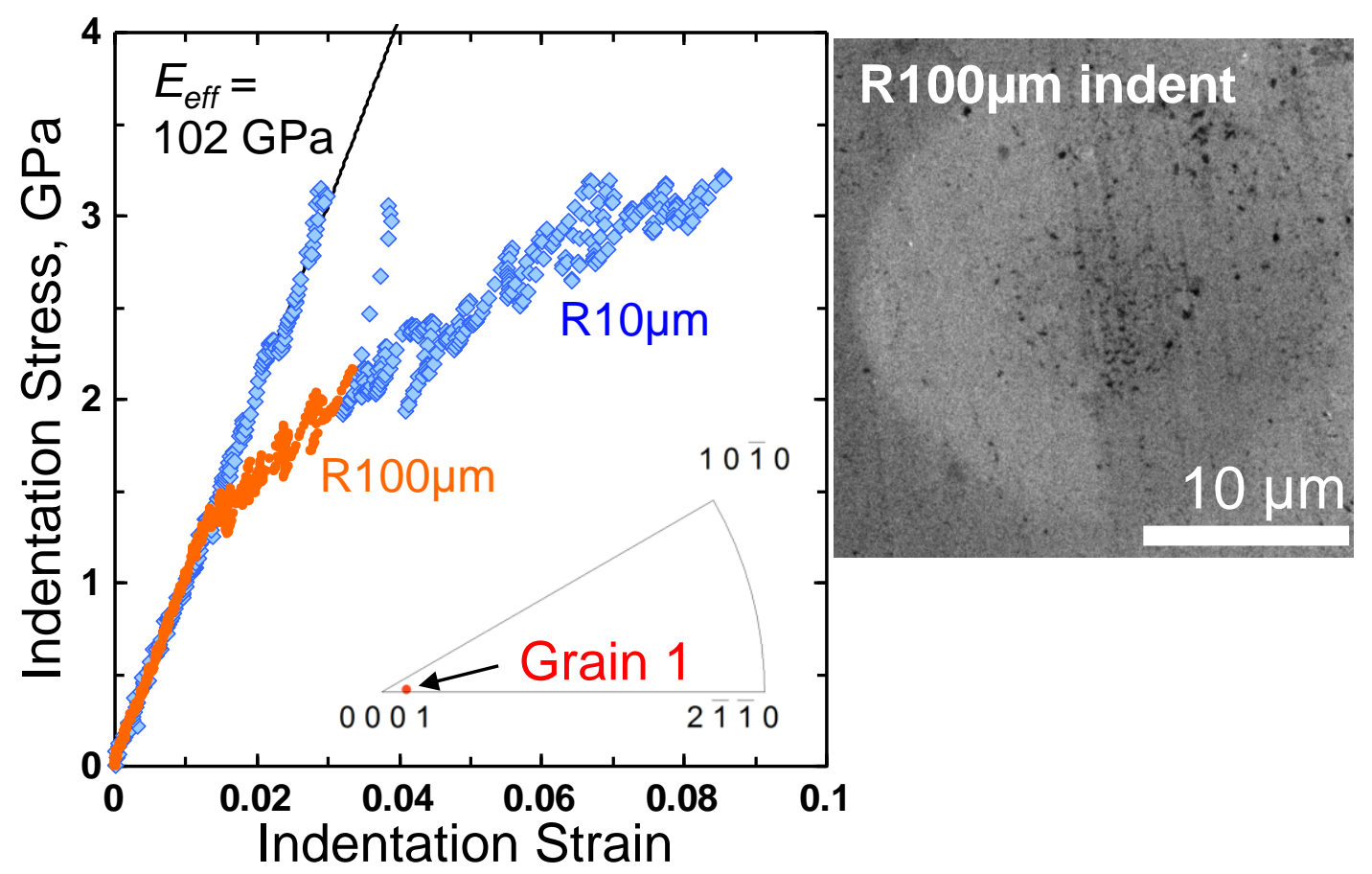

Figure 2. Spherical nano-indentation stress strain response of Grain 1, measured with two indenters of radii 10 and $100 \mu \mathrm{m}$. Inset. Orientation of Grain 1 on the IPF triangle. Right image: residual imprint from a 100 $\mu \mathrm{m}$ radius indenter on Grain 1. 

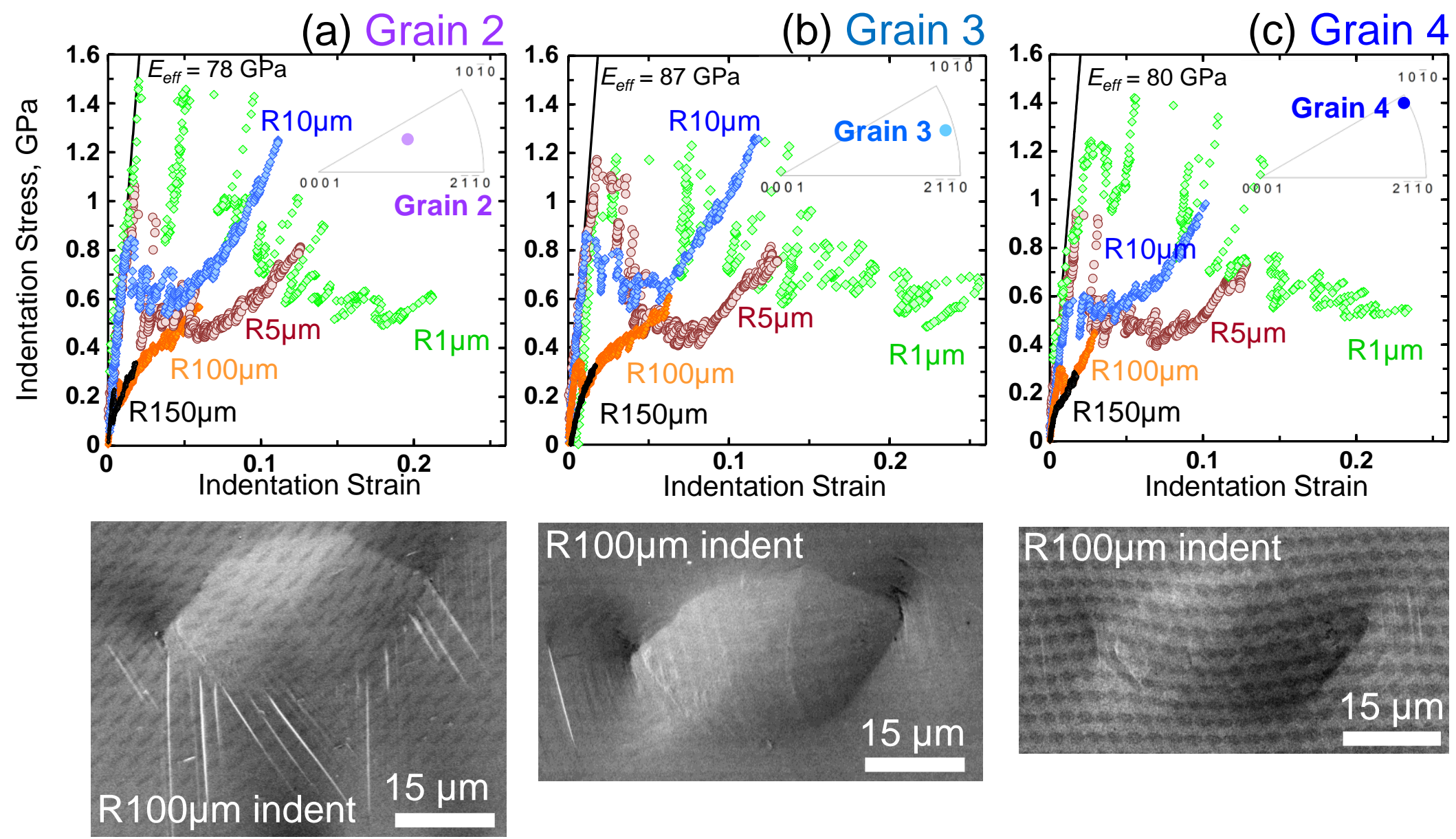

Figure 3. Spherical nano-indentation stress strain responses of (a) Grain 2, (b) Grain 3 and (c) Grain 4. Results from five different indenters of radii 1, 5, 10,100 and $150 \mu \mathrm{m}$ are shown for each case. The insets show the orientations of the respective grains on the IPF triangle. The SEM micrographs show the residual imprints from a $100 \mu \mathrm{m}$ radius indenter on each grain. The micrographs were taken at a $52^{\circ}$ tilt angle, and are shown here after correcting for the tilt angle. Due to shadowing effects caused by the high tilt angle, the material pile-ups on the left and right sides of the indents appear as darker regions in the micrographs; the deepest regions of the indent are towards the center of the micrographs. The dotted markings on the sample surfaces are an artifact from the EBSD scanning pattern. 
Table 1. Comparison of the indentation loading modulus $\left(E_{\text {eff }}\right)$, the indentation yield $\left(Y_{\text {ind }}\right)$, and hardening rates between the four grain orientations in $\mathrm{Zr}$, and fcc Al [19] and bcc Ta [45].

\begin{tabular}{|l|l|l|l|l|}
\hline & $\begin{array}{l}\text { Loading } \\
\text { modulus, } \\
\boldsymbol{E}_{\text {effs }} \text { GPa }\end{array}$ & $\begin{array}{l}\mathbf{0 . 2 \%} \text { offset } \\
\boldsymbol{Y}_{\text {inds }} \mathrm{MPa}\end{array}$ & $\begin{array}{l}\text { Hardening } \\
\text { rate, GPa }\end{array}$ & $\begin{array}{l}\text { Hardening } \\
\text { rate/Shear } \\
\text { modulus }\end{array}$ \\
\hline Zr Grain 1 & $101.6 \pm 6.7$ & $1279.7 \pm 151$ & $37.8 \pm 8$ & 1.04 \\
\hline Zr Grain 2 & $78.1 \pm 2.1$ & $107.4 \pm 11$ & $11.1 \pm 0.8$ & 0.3 \\
\hline Zr Grain 3 & $87.2 \pm 2.2$ & $115.7 \pm 23$ & $10.3 \pm 0.4$ & 0.28 \\
\hline Zr Grain 4 & $80.1 \pm 2.4$ & $113.1 \pm 11$ & $11.2 \pm 0.7$ & 0.3 \\
\hline Aluminum & 70 & & $52.2 \pm 3$ & 0.19 \\
\hline Tantalum & 142.3 & $308.7 \pm 5$ & 12.6 & 0.18 \\
\hline
\end{tabular}


Table 2. Comparison of the contact radius ( $a$ ) values measured for Grains 2, 3 and 4 for the five different indenter radii.

\begin{tabular}{|c|c|c|c|c|}
\hline & & Grain 2 & Grain 3 & Grain 4 \\
\hline $\mathrm{R} 1 \mu \mathrm{m}$ & $\begin{array}{l}a \text { at the end } \\
\text { of test }\end{array}$ & $609 \pm 3 \mathrm{~nm}$ & $541 \pm 29 \mathrm{~nm}$ & $560 \pm 8 \mathrm{~nm}$ \\
\hline $\mathrm{R} 5 \mu \mathrm{m}$ & \multirow{2}{*}{$\begin{array}{l}a \text { at start of } \\
\text { hardening }\end{array}$} & $859 \pm 86 \mathrm{~nm}$ & $979 \pm 140 \mathrm{~nm}$ & $1043 \pm 97 \mathrm{~nm}$ \\
\hline R10 $\mu \mathrm{m}$ & & $714 \pm 77 \mathrm{~nm}$ & $643 \pm 49 \mathrm{~nm}$ & $817 \pm 113 \mathrm{~nm}$ \\
\hline $\begin{array}{l}\text { R100 } \mu \mathrm{m}, \\
\mathrm{R} 150 \mu \mathrm{m}\end{array}$ & $a$ at yield & $1321 \pm 174 \mathrm{~nm}$ & $1513 \pm 521 \mathrm{~nm}$ & $1597 \pm 179 \mathrm{~nm}$ \\
\hline
\end{tabular}

